\title{
Flow In a Circular Pipe Filled With \\ Porous Medium in the non-Darcian Effects
}

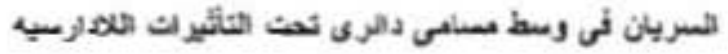

\section{M.S. El-Kady, M.A. Tolba and L.H. Rabie}

\section{Power Mechanical Engineering Departmeat} Mansoura University. Egypt

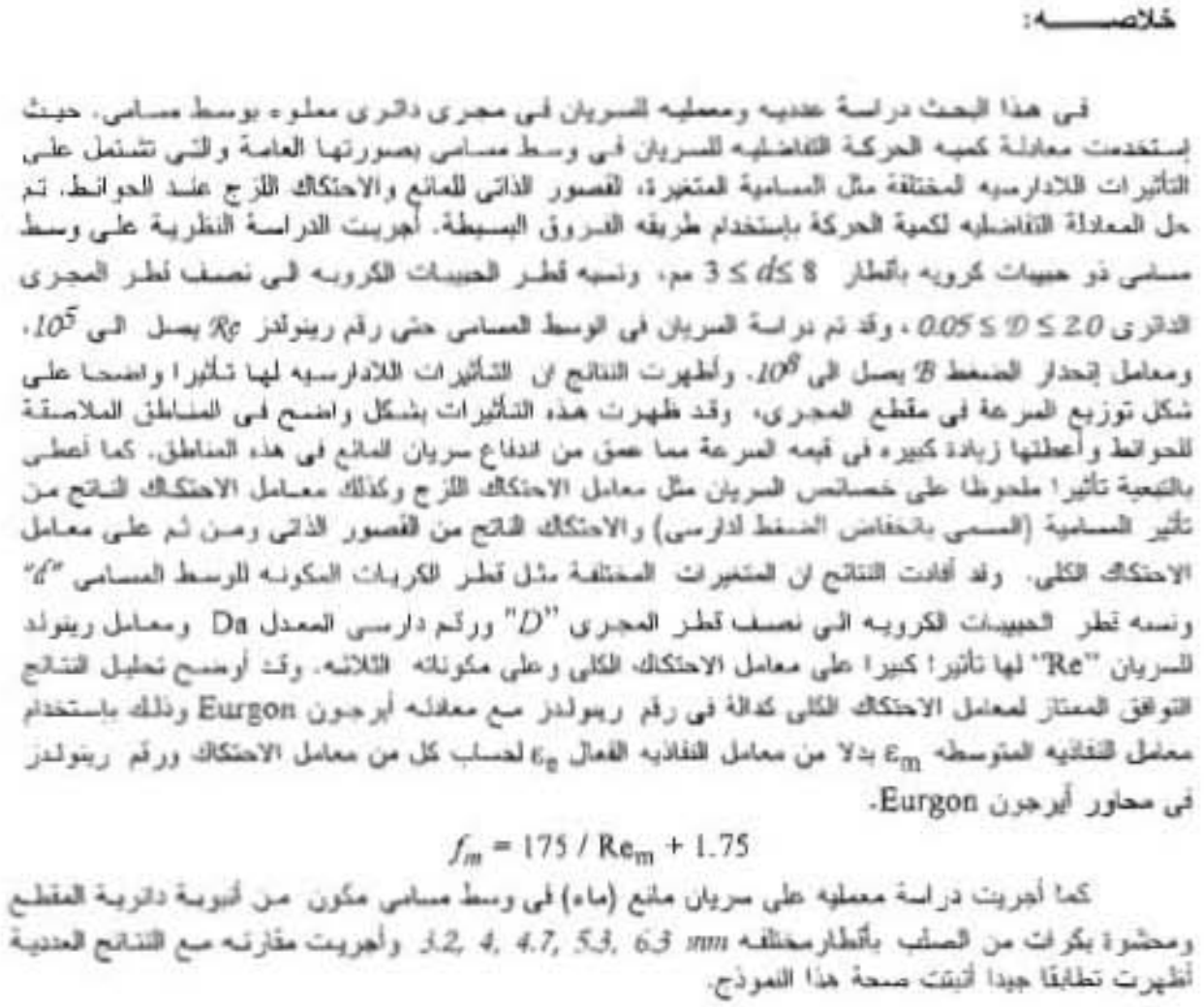

$$
f_{m}=175 / \mathrm{Re}_{\mathrm{m}}+1.75
$$

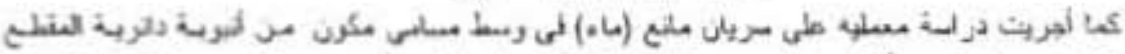

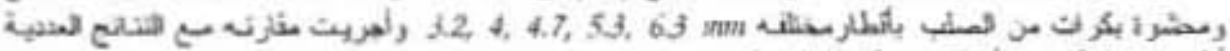

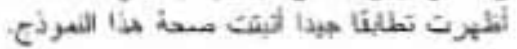

\section{Abstract:}

Forced flow through a circular pipe filled with saturated porous tnedia has been vumerically simulated. The generalized form of the momentum equation including the non-Darcias effects such as the vanable porosity, flow inertia, and viscous friction is considered. To solve the momentum equation, the finite difference method is used. Different sphere diameters of $3 \leq d \leq 8 \mathrm{~mm}$, and spbere diameter to the pipe radias ratio 
" $D "$ in the rango $0.05 \leq D \leq 2$ are considered. The results are obtained for flow Reynoids number up to $10^{5}$, and nondimensional pressure gradient $\mathrm{B}$ up to $10^{8}$. The results show that the non-Dareian effects have a significant influence on the velocity profiles. These effects appear clearily in the regions near to the wall and gives an increase in the magninade of the velocity and signifies the clanneling effect. It has in turns a significast influeace on the fluid flow characteristics such as the boundary frictional drag, the frictional drag induced by the solid matrix (designed as Darcy's pressure drop) and the flow inertia drag and in turns in the total drag coefficient. The results show the great influence of sphere diameter $d$, sphere diameter to the pipe radius ratio $D$, Darcy number Da and the Rcynoids number on the tocal drag coefficient $f_{f}$ and also on the behavior of its three componests. The predicted results of the total friction as function of Reynoids number exhibit excellent agreement with Erguat equation based on the area mean porosity $\varepsilon_{m}$ instead of the free porosity $\varepsilon_{c}$ It confirms the fact that Ergun equation is also valid for porous media of ideutical spherical particles for the laminar, transient and turbulent regimes.

$$
f_{m}=175 / \mathrm{Re}_{\mathrm{m}}+1.75
$$

To verify the oumerical results, an experimental investigation was carried out for the flow of water in a circular tube filled with five different steel spheres of diameters $3.2,4,4.7,5.4$ and $6.3 \mathrm{~mm}$ respectively. Comparison with predicted results shows a very good agreemeat, and proves the validity of the model.

\section{Iotroduction}

Fuid flow in a porous media bas been of continuous interest for the past five decades. This interest stems from the complicated phenomena associated with the flow process in porous media, and its very wide applications available. Such applicatious can be found in agriculture, chenvical engineering, environmental protection, material scieace, thermal insulation, grain and coal storage, underground water hydrology, soil mechanios, drying techuology, transpiratiou cooling. solid matrix beat exchanger. ceramic processing, catalytic reactors, and food processing technology. Consequently. understanding the associated transport processes is of critical importance.

The majority of the existing studies are pertinent to the fluid flow and heat traisfer in porous media based on the Darcy tlow model. The theoretical work of Vafia and Tien [1] attempted to account for boundary and isertial effects on forced convective fiow in porous media. Vafia [2] and $\mathrm{Vafa}$ et al. [3] studied the effects of flow clunneling on forced convection aiong a flat plate theoretically and experimentally. Kaviany [4] investigated a lamisar flow through poroas medium bounded by isothermal parallel plates with the Brinkman-extended flow model and constant matrix porosity and Nakayama et al. [S] treated the wall by a constant beat flux and peripherally uniform wall temperature, Polikakos and Renken [6] analyzed theoretically the forced flow in a channel filled with porous medium, and accounted for the effects of flow inertia, variable porosity, and Brinkman friction. But they did not 
study these effects on the fluid flow characteristics. El Kady [7] investigated theoretically the forced convection heat transfer and flow in an annular channel filled with porous media taking into consideration the noa Darcian effects. Cheng, et al. [8] analyzed the forced convection in the entrance regios of a packed channel with asymmetric beating. The variable porosity close to an impermeable boundary has been reported by a number of investigators such as Beaenati and Brosilow [9] They show a distinct porosity variation in packed beds. Their results show a high porosity region close to the external boundary. Chandrasekhara and Vortmeyer [10] used the measureweots of Benenati and Brosilow [9] to solve numerically for the velocity profile in isothermal packed beds. Wang and Du [11] analyzed experimentally the forced convective beat transfer in a vertical annulus filled with porous media. Amiri and Vafia [12] simulate aumerically the forced coavective incormpressible flow through porous media, and the associated transport processes.

The existing literature on the fluid Dow through porous media (packed sphere beds), give very limited quantitative information on the influence of considering the noo-Darcian effects such as the variable porosiry, Dlow inertia, and viscous friction on the fluid flow characteristics. It is the objective of this paper to provide an analysis for the flow through a circular pipe filled with saturated porous media (packed sphere beds), taking into coesideration the variable porosity, flow inertia, and viscous friction and see its significant influence on the Duid Dow characteristics such as the boundary frictional drag, the bulk frictional drag induced by the sobid matrix (designed as Darcy's pressure drop) and the flow inertia drag induced by the solid matrix at bigh flow rates (designed as Forschheimer's form drag).

\section{Mathematical Formulation}

In order to formulate the problem, a steady, hydrodyaamically fully developed fluid flow in a horizontal circular pipe filled with packed spleres as a porous medium is considered $\mathrm{It}$ is assumed that the luid and the solid matrix are in local thernal equilibrium and that the maguindes of the physical properties such as the viscosity and deesity are constant. The physical configuration of the problem is strowa in Figure (1).

Under these assumptions and by treating the solid matrix and the fluid as a continuum, the improved Darcy momenrum equation including the viscons boundary friction and inertia effects are used:

$$
1 / \rho \cdot[\partial \mathrm{P} / \partial \mathrm{x}]=v / \mathrm{r}[\partial / \partial \mathrm{r}(\mathrm{r} \partial \mathrm{u} / \partial \mathrm{r})]-v u / \gamma \cdot \mathrm{A} u^{2}
$$

where, $u, P, P, u$ are the velocity in the axial direction $x$, the pressure, the fluid density and the fluid dyamic viscosity respectively. $Y$ and $A$ are the permeability and the inertia coefficient (Forschheimer function) of the porous medium and are dependent on the porosity $\varepsilon$ and otber geometrical parameters of the medium. These parameters 
are given by Ergua [13] for backed beds of ideatical spherical beads of diameter $d$ and porosity $\varepsilon$ as:

$$
\begin{aligned}
& y=d^{2} \varepsilon^{3} /\left[175(1-\varepsilon)^{2}\right] \\
& \mathrm{A}=1.75(1-\varepsilon) /\left[d \varepsilon^{3}\right]
\end{aligned}
$$

The presest approach using equation (1) can be used from $y \rightarrow 0$ (Darcian flow) to $\gamma \rightarrow \infty$ (Pure fluid tlow). The first, second, and third terms on the right hand side of oquation (1) are expressions for the boundary viscous drag which was introduced first by Brinkman [14], Darcy frictional drag which is responsible for the porous structure and inertia drag.

Due to the fact that, the particle diameter to the pipe radius ratio " $D$ " is large eaough, the porosity variatiou ueat the wall in no longer neglighle. Therefore, it is assumed that the porosity " $\varepsilon$ " varies exponentially from the wall The experimental results of Benenati and Brosilow [9] for the porosiry variation which is used tater by Vafia et.al [3], Poulikakos and Renkeu [6], and Mularidhar and Kulacki [15] can be represented in the following form:

$$
\varepsilon=\varepsilon_{e}[1+h \exp (c, y / d)]
$$

Where $y$ is the distance from the boundary wall, $\varepsilon_{e}$ is the free stream porosity, and the empirical coustants $\mathrm{b}$ and $\mathrm{c}$ are dependent on the particle diameter.

Numerical predictions were camied out for a porous media forned by spheres of diameter 3, 5, 6 and $8 \mathrm{~mm}$. The constauts chosen to represent the variation of $\varepsilon_{e}, b$, and $c$ are similar to that used by Beneoati and Brosilow [9] and Chandrasekhara and Vortmeyer [10] and El kady [7] umong others. In the cases of $d=3,5$ mum the values of the parameters are $\varepsilon_{e}=0.37, b=0.35$ and 0.43 and $c=3$. For $d=8 \mathrm{~mm}$, in the other hand, the values of the parameters are $\varepsilon_{e}=0.37, b=0.9$ and $c=2$. For the case of $d=$ $6 \mathrm{~mm}$ the following values are assumed $\varepsilon_{e}=0.37, b=0.53$ and $c=3$.

The boundary conditious imposed on the physicai systern are uniform with respect to the axial coordinate, the computational domain thus comprises of one balf of the pipe over which the velociry $u=0$ at $r=r_{0}$

Using the dimeasioniess variables $\mathrm{U}=\mathrm{u} / \mathrm{u}_{\mathrm{m}}, \mathrm{R}=\mathrm{r} / \mathrm{r}_{\mathrm{o}}$ and $D=d / \mathrm{r}_{\mathrm{o}}$, the momentum equation ( 1 ) can be transformed to nondimensional form as:

$$
U+C_{1} \cdot[R e / 2] \cdot U^{2}=(B \cdot \Gamma) \cdot[2 / R e]+(\Gamma / R) \cdot[\partial / \partial R(R \partial U: \partial R)]
$$

where, $C_{1}=0.01 D /(1-\varepsilon)$.

$$
\begin{aligned}
& r=\gamma / r_{0}^{2}=D^{2} \varepsilon^{3} /\left[175(1-\varepsilon)^{2}\right], \\
& R e=2 u_{m} \cdot r_{d} u, \text { and }
\end{aligned}
$$




$$
B=-\partial P / \partial x \cdot\left[r_{0}{ }^{3 / \rho} \rho u^{2}\right]
$$

$u_{m}$ is the averaged fluid velocity inciuding the solid and fuid regions and $B$ is the noedimensional pressure gradient.

\section{Fluid Flow Characteristics}

The boundary frictional drag represents ab overail characteristics of the channel flow, it is determined by the friction coefficieot which is defined as [16-17] ;

$$
\varepsilon_{v}=\tau_{w} /\left[1 / 2, \rho u_{r}^{2}\right]
$$

where $\tau_{w}$ is the mean wall shear stress and

$\mathrm{u}_{\mathrm{f}}$ is the overage local average velocity in the $\mathrm{x}$-direction in void volume

In addition to the boundary frictional drag, the flow through the porous duct experiences also a bulk frictional drag induced by the solid matrix (designed as Darcy's pressure drop) and a flow inertia drag induced by the solid matrix at high flow rate (designed as Forschheimer's form drag). The bulk friction (Darcy) and the mertia (Forschheimer) drag coefficient and the total bulk drag which is the summation of the three drag rypes are defined [16-17] after changing the variables to our notatious and definitions as follows:

$$
\begin{aligned}
& f_{D}=\mu \gamma_{\mathrm{m}}{ }^{-1} \varepsilon_{\mathrm{m}} u_{\mathrm{f}}\left(\mathrm{r}_{d} / 2\right) /\left[1 / 2 \rho \mathrm{ur}^{2}\right] \\
& f_{i}=0.143 \rho \gamma_{\mathrm{m}}{ }^{-0.5} \mathrm{~s}_{\mathrm{m}}{ }^{0.5} \mathrm{ur}^{2}\left(\mathrm{r}_{\mathrm{o}} / 2\right) /\left[1 / 2 \rho \mathrm{ur}^{2}\right] \\
& f_{t}=-(\mathrm{dP} / \mathrm{d} \mathrm{x}) \cdot\left(\mathrm{r}_{0} / 2\right) /\left[1 / 2 . \rho \mathrm{uf}_{\mathrm{f}}{ }^{2}\right]
\end{aligned}
$$

where $\gamma_{m}$ is the permeability based on the area mean porosity $\varepsilon_{m}$

$$
\varepsilon_{m} \text { is the area mean porosity } \varepsilon_{m}=\left[1 / r_{0}{ }^{2}\right]-\int_{0}^{r_{0}} 2 r c d r
$$

Equations (6) - (9) can be written as a function of the nondimensional parameters as:

$$
\begin{aligned}
& f_{v}=\left.4(\mathrm{dU} / \mathrm{dR})\right|_{\mathrm{ro}} / \mathrm{Re}_{q} \\
& f_{D}=1 / 2 \cdot \mathrm{Da}^{-1} / \mathrm{Re}_{\mathrm{f}} \\
& f_{i}=0.0715 \mathrm{Da}^{-0.5} \\
& f_{t}=f_{v}+f_{D}+f_{i}=4 \mathrm{~B} / \mathrm{Re}_{\mathrm{q}}{ }^{2}
\end{aligned}
$$

where $\quad \mathrm{Da}$ is the modified Darcy number $=1 \mathrm{~m} /\left(4 \mathrm{r}_{\mathrm{o}}{ }^{2} \mathrm{E}_{\mathrm{m}}\right)$

$R_{e f}$ is the Reynolds number based of the velocity $u_{f}, R_{e q}=2 u_{f} r_{d} / u$

In the present study equations $(10) \cdot(13)$ are used. 


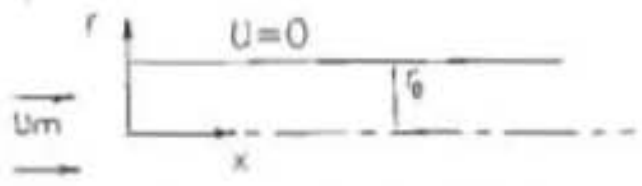

Fiz I Plysical model, Coordioate system and boundaries

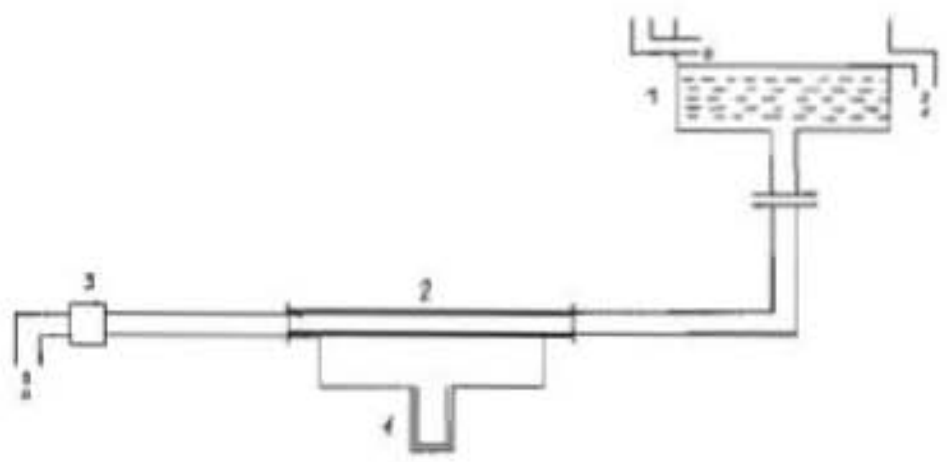
1. Overtsead tunk
2. Test section
3. Flow metet
4. U-tube masometer

Fig. 2 Schematic diagram of the experimental apparatus 


\section{Method of Solution}

Finite difference equation is derived from equation (5). Both the first and second order derivatives were discretized by using ceatral difference formulas [18]. The Forschheimer nonlinear term is linearized by guessing initial values of the veiociry field at all the grid points, and the nonlinear terw was written as the product of the unknown veiociry and the guessed velocity. The difference algebraic equation is solved using the Gauss-elimination metbod [19] to yield the velocity feld. A variable grid for accurate resolution of the important near-wail region is used in the $\mathrm{Y}$-direction to obtain the momentum equation faite difference form.

\section{The Experimental Work:}

To validate the program developed for this study an experimental study for the flow in a porous media is carried out. A scbematic diagram of the test rig is shown in Fig. 2. The test section is made of a copper tube of $20 \mathrm{~mm}$ inside diameter, and a 250 $\mathrm{mm}$ long. The tube is filled uniformly with stainless steel splaeres of uniform sizes to form a backed bed to serve as the solid porous matrix through which fluid flows. The steel spheres are held in place by means of wire mesh located at the two ends of the tube. Five different sphere diameters are used through out this work. They are 3.2, 4.0, $4.7,5.4$ and $6.3 \mathrm{~mm}$ in diameter respectively. Fluid flows under the gravity action from a constant head tank $6.0 \mathrm{~m}$ above the test section. The porous section is kept horizontal in the gravitational open flow system. A calibrated orifice-meter is used as a llowmeter to measure the flow rate and from which the mean velocity and Reynolds number of the flow were calcuinted. A U-tube mercury manometer is connected to two pressure taps located just upsuream and downstream the test section to measure the pressure drop along the test section. During the experiments the flow rate varied from $9.42 \times 10^{-6}$ to $166 \times 10^{-6} \mathrm{in}^{3} / \mathrm{s}$. The experimeats were performed carefilly, each time the experiments were initiated at the largest flow rates; the objective was to produce a stable packing of the beads and to prevent the effects of changing porosity on the pressure drop as the flow rate was varied.

\section{Results and Discussion}

\subsection{Flow velocity and Chaneling}

The Velocity distribution across the pipe flow in a porous media exhibit a cbanneling effect near the wail. It is considered the main driver for the behavior of the flow characteristics in such Dow types. In order to give good explanations for the effect of the different geometric parameters such as the bead diameter $d$ and its ratio to the pipe radius $D$ on the flow characteristics, the velocity distribution and the channeling effect were studied. 
H. 8 Dr, M.S. E1-Yady, Dr. H.A. Tolba and Dr,L.H, Rabie

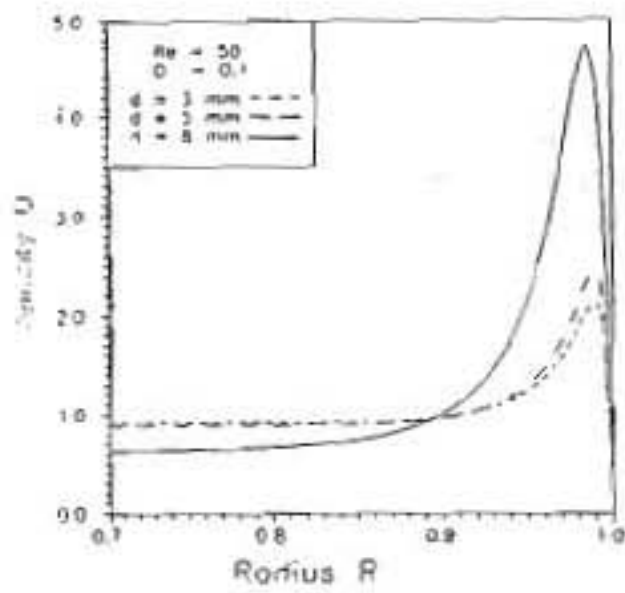

Fie 3 Veiocity distribution across the chrunel talf width for differeat values of bead diameter of, $D=0.1$ and $\mathrm{Re}_{\mathrm{e}}=50$

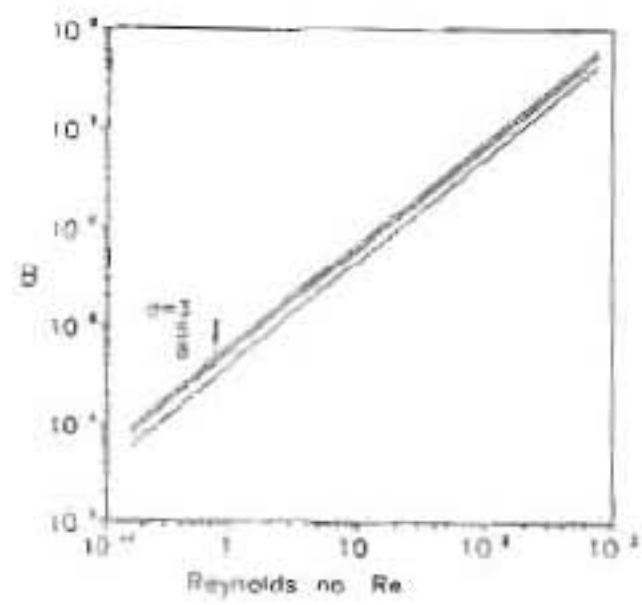

Fig 5 Dependence of the pressute drop B or $\mathrm{Re}$ for different values of bead disineter $d$ and $D=0.1$

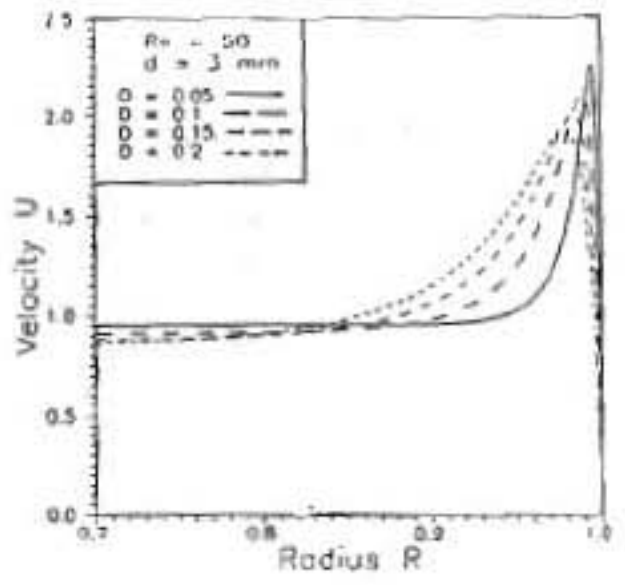

Fig. 4 Flow Velocity distribution across the chanel late width for different values of $D, d=3 \mathrm{~mm}$ aud $\mathrm{Re}=50$

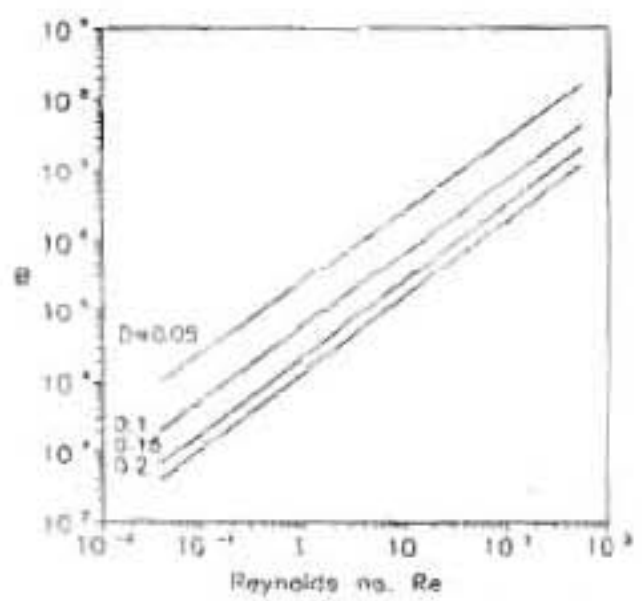

Fig 6 Dependence of $B$ on Re for a host values of beand diameter to channel radius $D$ and $d=3 \mathrm{~mm}$ 
The depeadence of tbe flow velociry and tbe chanaeling effect across the pipe half section oo the bead diameter $d$ and its ratio to the cbaninel radius $D$ are shown in Figs. 3 and 4 . Fig. 3 shows the velocity distribution for the flow in a porous media of different sphere diameters $d=3,5$, and $8 \mathrm{~mm}, D=0.1$ and $R_{c}=50$. Fig. 4 shows the velocity distnbution for the dow in porous anedia of different spbere to pipe diameters ratio $D(0.05 \leq D \leq 2), d=3 \mathrm{~mm}$ and $\mathrm{Re}=50$. Both Figures show that with the increase of either $d$ or $D$ the permeability aear the wall increases whicb in turns iscreases the channeling flow area aear the wall.

\subsection{Fow characteristies}

The Ouid tow is characterized by the friction factors $/ \mathrm{m} / \mathrm{d}_{\mathrm{h}} f_{\mathrm{t}}$ and $f_{\mathrm{t}}$ which ore described by equations $(10-13)$

The total friction factor $f_{t}$ depends mainly on both Reypolds number and the noedimensional pressure gradient B. To show the effect of the different geometrical parameters on $f_{t}$ it is very useful to know first the relation berween both Re and $B$. Figs. 5 and 6 sbow the behavior of the nondimensional pressure gradient $B$ with the change of Reynolds uumber for different values of $d$ and $D$. Both Figures show the lanear relation between $B$ and $R e$ in the logaritumic scale. The results sbow an increase in B with the increase of Re and its decrease with the increase of either the bead diameter $d$ of the ratio $D$ at constant Reyoolds number.

Fig 7 represeats the variation of the total friction factor $f$ with $\operatorname{Re}$ for $d=3,5$ and 8 $\mathrm{mm}$ at cotstat $D=0.25$. The total friction $f_{l}$ decteases with the increase of the bead diameter. For constant Reyoolds number the pressure gradieat $B$ decreases with the nerease of the bead diameter as stown in Fig. 6 which leads to the increase of $/ t$. Fig 8 shows the variation of the total friction factor $f$, with Re for a range of the bead dianeter to the tube radius ratio $0.05 \leq D \leq 1$. With the increase of $D$ the aoudimensional pressure gradient B decreases as shown ia Fig. 6 which yields the decrease of the total fiction factor $f_{1}$ with the increase of Re and $D$. Lu Figs 7 and 8 , the $\left(f_{r}\right.$ Re) curves take the limearly shape in the laminar region, curved sbape in the trassient and constant value for the turbuleat regions.

The behavior of the boundary viscous drag $f_{v}$, the Darcy friction $f_{D}$, incria fiction $f$ and the total drag $/$ with Reyoolds number Ref are presented in fig. 9 for $d=$ $3 \mathrm{~mm}, D=0.1$ and ia $\mathrm{fg}_{\mathrm{g}} .10$ for $d=5.4 \mathrm{~mm}$ and $D=0.54$. It is sluows in Fig. 9 that the inertia friction factor $f_{f}$ is independeat on Re, while the Darcy friction factor $f_{0}$ changes linearly in the logarithmic grapb with $R c_{f}$. The values of $f_{i}$ exceed the values of $f_{v}$ when $R_{e}>65$ and exceed the values of $f_{D}$ when $R e_{f}>300$. The curve of $f_{t}$ assymptotes with the lines of $f_{L}$ is the linearly shape laminat regioa and is assymptotes with the line of $f_{i}$ in the constant value region where $R_{e}>5 \times 10^{4}$. In fig. 10 where bigger void area exists for the case of $d=5.4$ and $D=0.54$, the same trend of the data of the friction factors is observed but with smaller values 
M.10 Dr, M,S, E1-Kady, Dr, M.A. Tolba and Dr, L.H. tobin

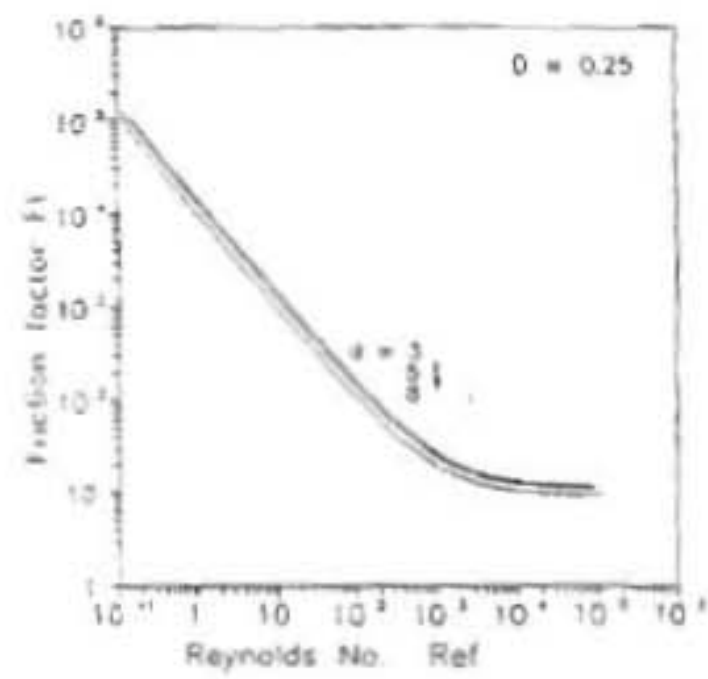

Fig. 7 total friction factor $f t$ variation wich $R e_{\mathrm{f}}$ for $d=3,5$ and 8 mm and $D=0.25$

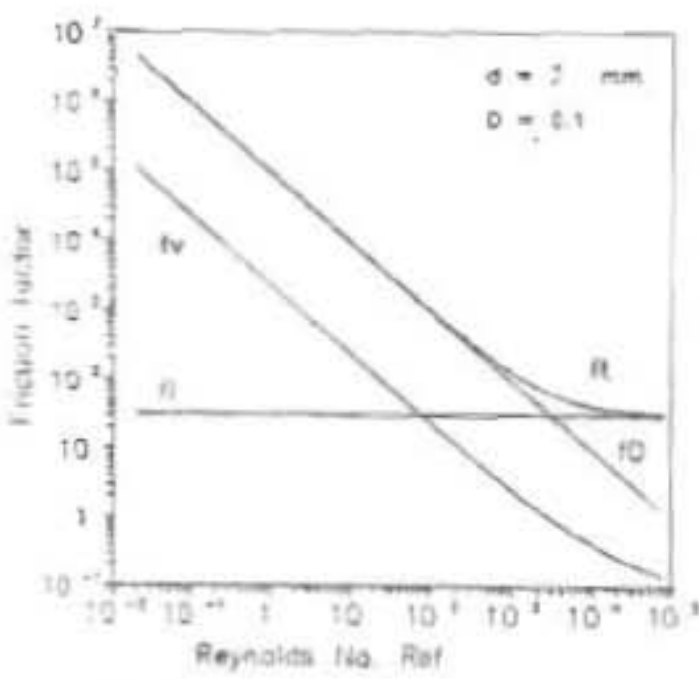

Fig 9 friction factors $f_{t}, f_{D}, f_{v}, f_{i}$ variation with $R_{e}$ for $d=3 \mathrm{~mm}$ and $b=0.1$

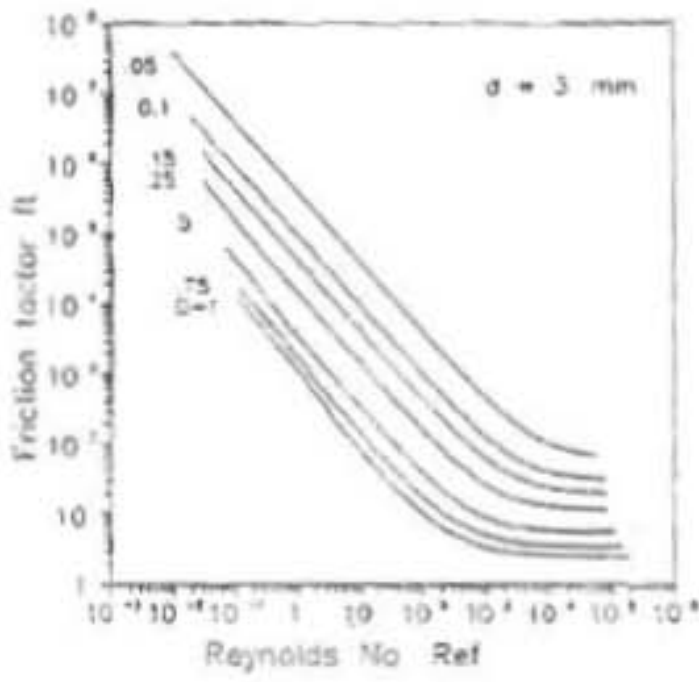

Fig. 8 total friction facter $f_{t}$ variation with $\mathrm{Re}_{\mathrm{f}}$ for a bost of $\mathrm{D}$ values and $\mathrm{d}=3 \mathrm{~mm}$

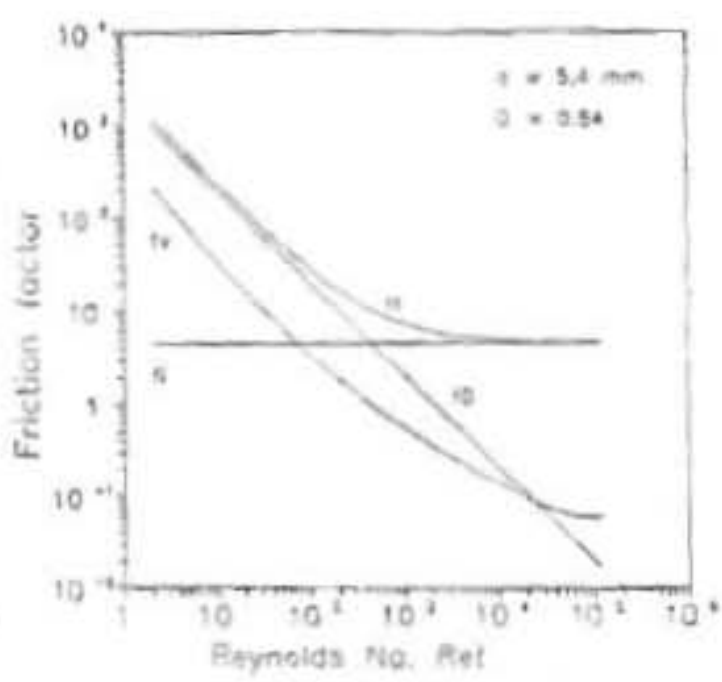

Fig 10 friction factors $f_{l}, f_{0}, f_{v}, f_{1}$ variation with $R_{e}$ for $d=5.4 \mathrm{~mm}$ and $D=0.54$ 
Fig. II presents the relative sharing of each of the different friction factors in the total friction. It represents the values of $f_{D} / f_{t}, f_{v} / f_{t}$ and $f_{i} f_{t}$ for $d=3 \mathrm{~mm}$ and $D=$ $0.1,0.25$ and 0.5 . With the increase of $D$ i.e. the increase of the void volume, the values of the Darcy friction $f_{D} f_{I}$ decreases while both the inertia and viscous friction factors $f_{v} / f_{t}$ and $f_{i} f_{t}$ increases. For $R_{e_{1}}>5 \times 10^{4}$ both the Darcy and viscous friction $f_{D} / f_{t} . f_{V} / f_{t}$ can be neglected, while the inertia friction $f / f_{t}$ forms the main part of the total friction. For $\mathrm{Re}_{\mathrm{f}}<100$ both the friction factors $f_{V} / f_{t}$ and $f_{f} / f_{t}$ are nearly constants but the Darcy friction $f_{D} / f_{t}$ coutributes the main part of the total friction. Fig. 12 gives the sane relations showa in Fig. 11 but for more void media, for $d=5.4 \mathrm{~mm}$ and $D=0.54$. The same trend appears witb higher values of $f_{v} / f_{t}$ and $f_{i} / f_{t}$ but the Darcy friction $f_{D} / f$, takes swaller values thas that of the dense porous media presented in Fig. II.

Fig. 13 illustrates the effect of Darcy number on the product of friction factor and Reynolds number. The total friction factor $f_{t} \mathrm{Re}_{\mathrm{f}}$, boundary friction $f_{v} \mathrm{Re}$, Darcy's friction factor $f_{D} \mathrm{Re}_{4}$, and Forschbeimer's friction factor $f_{i} \mathrm{Re}_{4}$, are plotted over the range $60 \leq \mathrm{Da}^{-1} \leq 10^{6}$. It is seen that the value of $f_{D} R_{e}$ exceeds $f_{V} R e_{f}$ when the value $\mathrm{Da}^{-1}>120$, the curve of $f_{t} \mathrm{Re}_{\mathrm{f}}$ overlaps with the lines of $f_{D} \mathrm{Re}_{\text {f }}$ for $\mathrm{Da}^{-1}>$ $5 \times 10^{4}$ For $\mathrm{Da}^{-1} \leq 1$ Darcy friction $f_{D} \mathrm{Re}_{1}$ and Forschbeimer's inertia friction factor $f_{i} \mathrm{Re}_{f}$ are almost oegligible and the flow behaves just like the Posseuille flow. The results of parallel plates of Nakayama et al [5] and of the square channel of Hwang et al [17] are also represented with these results in Fig. 13 for comparison. A similar trend is observed between the data of parallel plates, the square channel and the circulat channel.

It is tnore convenient to cast one relation to include the effects of the porosity variation and the sphere diameter. So, it is more suitable to plot the total friction factor versus Reynolds number in the nondimentional Ergun coordinates. In these coordinates the total friction factor and Reyzolds number are based on the sphere diameter $d$ and the free stream porosity $\mathrm{E}_{\mathrm{e}}$ and are defined as

$$
\begin{aligned}
& f_{f}=(\mathrm{dP} / \mathrm{dx})(d / 2)\left[\varepsilon_{\mathrm{e}}{ }^{3} /\left(1-\varepsilon_{\mathrm{e}}\right)\right] /\left[1 / 2, \rho u_{m}{ }^{2}\right] \\
& \mathrm{Re}_{d}=u_{m} d / v \cdot\left[1 /\left(1-\varepsilon_{\mathrm{e}}\right)\right]
\end{aligned}
$$

The relation between $f_{e}$ aud $\mathrm{Re}_{d}$ is known as Ergun equation and represents the flow in laminar, transition and turbulent regimes. It fis available experimental data in tluid tlows very well, (Ergun [13] and Rabie al al [20]). It is used for beds of small randornly unspherical beads as:

$$
f_{e}=175 / \mathrm{Re}_{d}+1.75
$$

Figure 14 represents the relation of $\mathrm{f}_{\mathrm{e}}$ versus $\mathrm{Re}_{d}$ for $d=3 \mathrm{inm}$ and the different cases presented in Fig. 11 for $D=0.05,0.15,0.25,0.5,0.66,0.75$, and 1.0 . Figure 14 shows that such presentation of results made all the data to collapse on neariy one curve. Comparing these results with those reported by Ergun and represented by the 
M. 12 Dr. M.S.EL-Xady, Dr. M.A. Tolba and Dr. L.H. Rable

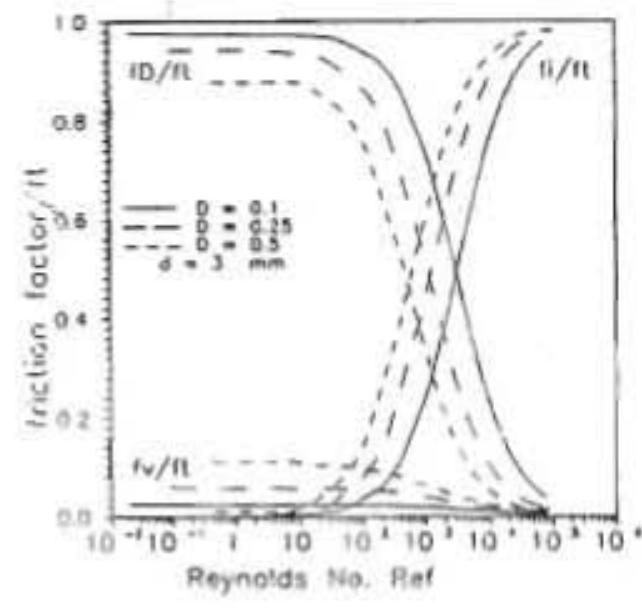

Fig. 11 friction factors $f_{D} f_{b}, f_{v} / f_{t}$ and $f_{f} f_{t}$ variation with $\operatorname{Re}_{\mathrm{f}}$ for $d=3 \mathrm{~mm}$ and $D=0.1$

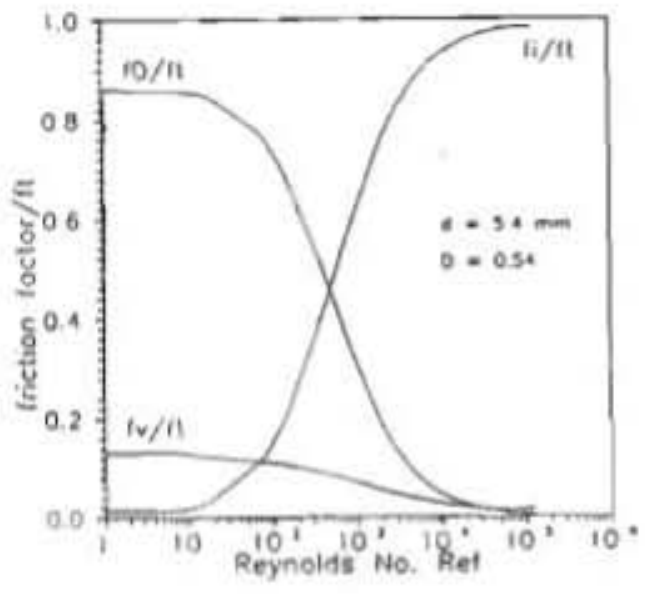

Fig. 12 friction betors $f_{D} f_{h} f_{V} f_{t}$ and fil $f_{t}$ vanation with Ref for $d=5.4 \mathrm{~mm}$ and $D=0.54$

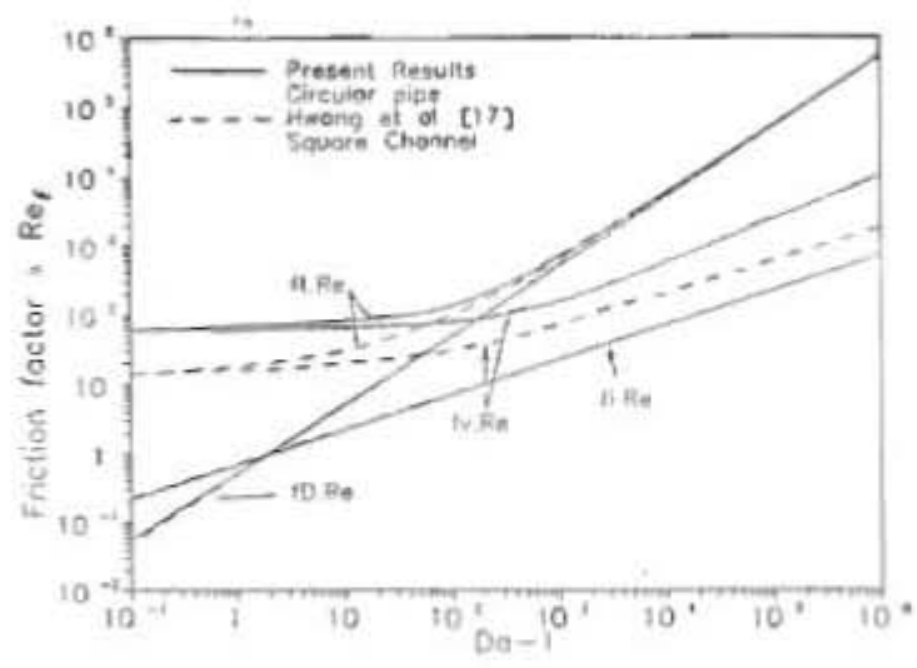

Fig. $13 f \mathrm{Re}_{\mathrm{f}}$ versas $\mathrm{Da}^{-1}$ for the present case and comparison with Hwang et at [17] for the square channel 
empincal formula (16) for the identical spberical beads sbows good agrecuent for the cases which have low values of the ratio of bead sphere diameter to the pipe outer radius, mainly $D=0.05$ and $D=0.15$. For higher values of $D$ the data deviates from the Ergun curve. The deviation increases with the increase of $D$. The reasoa for such deviation may be attributed the channeling effect which is caused by the porosity varation aear the wall and the higher velocity occurred there. For small values of $D$ the channeling effect is small and the free stream porosity $\varepsilon_{e}$ tends to equal the area mean porosity $\epsilon_{m}$, therefore, the deviation in this case is very small. By the increase of $D$ the porosiry vanation near the wall and the chaneling effects increase. Therefore, the free stream porosity $\varepsilon_{e}$ becomes more less than the area mean porosity $\varepsilon_{m}$ and the deviation increases

Detining $f_{m}$ and $\mathrm{Re}_{\mathrm{m}}$ as the total friction factor and Reypolds number based on the area mean porosity $\varepsilon_{\mathrm{m}}$ instead of the free porosity $\varepsilon_{\mathrm{s}}$ a modified form of Ergou equation for the identicai spherical beads can be casted from both variables $f_{m}$ and $\operatorname{Re}_{m}$

$$
f_{m}=175 / \mathrm{Re}_{\mathrm{m}}+1.75
$$

Using the previous definitions in equations (17) the data of Fig. i4 cas be represented in modified Ergun coordinates. Fig. Is presents the relation between $f_{m}$ vernus $R_{\mathrm{m}}$ for $d=3$ un and the different cases presented in Figs. 11 and 12 for $D$ $0.05,0.15,0.25,0.5,0.66,0.75$, and 1 . Fig. 16 presents the relation between $f_{m}$ versus $\operatorname{Re}_{\mathrm{m}}$ for $D=0.1$ and different bead diameters $d=3,5,6$ and $8 \mathrm{~mm}$. Such acw preseatation of resalts made all the data to collapse on one curve. This curve shows an excellent agreement with those results reported by Equation (17) for all the presented cases of $D$ and $d$ and for the laminar, uansient and rurbuleut regimes. This result confirms the fact that Ergun equation can also be valid for porous media of identical spherical particles but with Reyoolds number and the total friction factor based oa the area neaa porosity $\varepsilon_{\mathrm{m}}$ instead of the free porosity $\mathrm{c}_{\mathrm{e}}$

To validate the numencal model experimental test series were camed out. in which both the pressure drop and the volume tlow rate were recorded and both Reynolds number and the total friction factor based on the area mean porosity $\varepsilon_{m}$ were calculated and compared with the oumencal results. Fig. 17 preseats the comparison between the experimeatal and numericai values of the total friction factor with the clange of Reyoolds number for five spherical sized packed besds of 3.2,4, 4.7,5.4 and 63 mo diameter. The comparison shows good agreement of the presented results and proves the validiry of the model

\section{Conciusions}

In the preseat srudy, the generalized momentum differential equation including the non-Darcian effects such as the variable porosiry, flow incrtia and Brinkman viscous friction are solved by a numerical finite difference scheme to deternitice tbe flow 
N. 14 Dr. M.S. E-Kndy, Dr. M.A. Tolba and Dr. L.H. Rahze

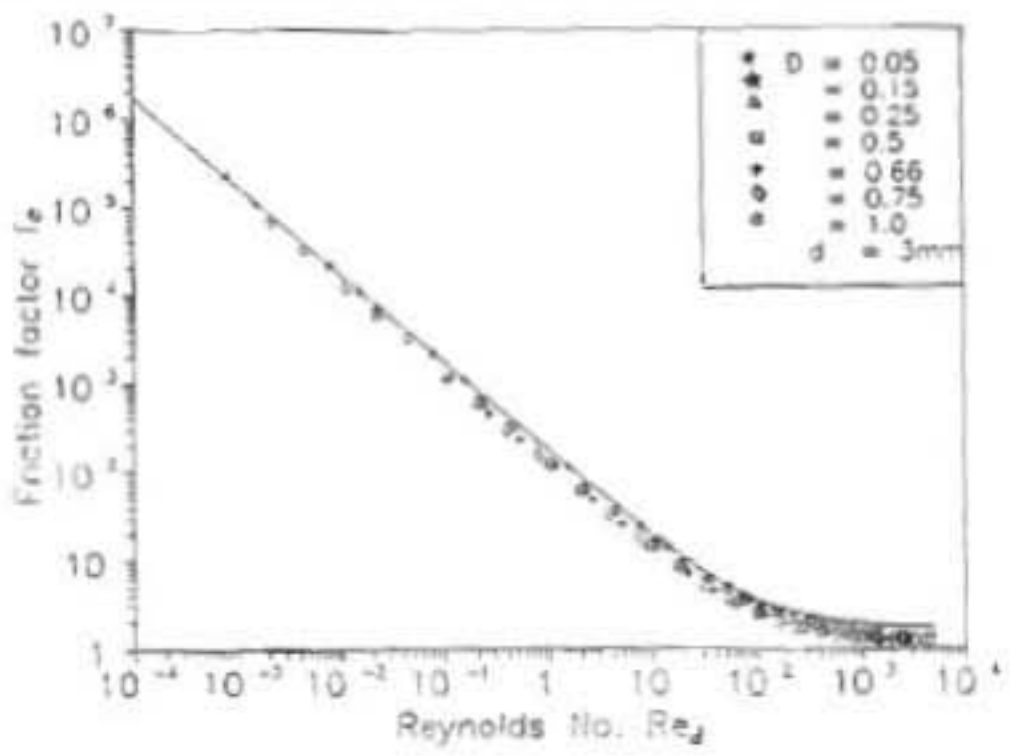

Fig 14 total fiction factor $f_{e}$ variaticn with Re for a bost of $D$ values and $d=3 \mathrm{~mm}$

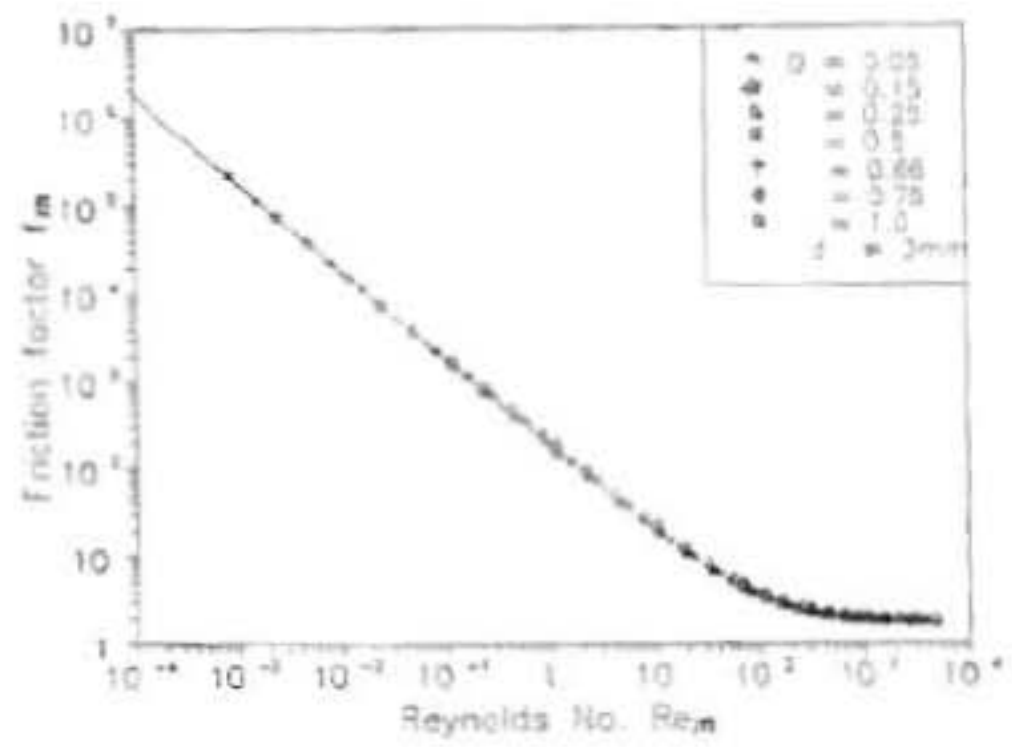

Fig. is cotal frictica factor $/ \mathrm{m}$ varation with $R \mathrm{e}_{\mathrm{w}}$ for a bost of $D$ values and $d=3 \mathrm{rom}$ 


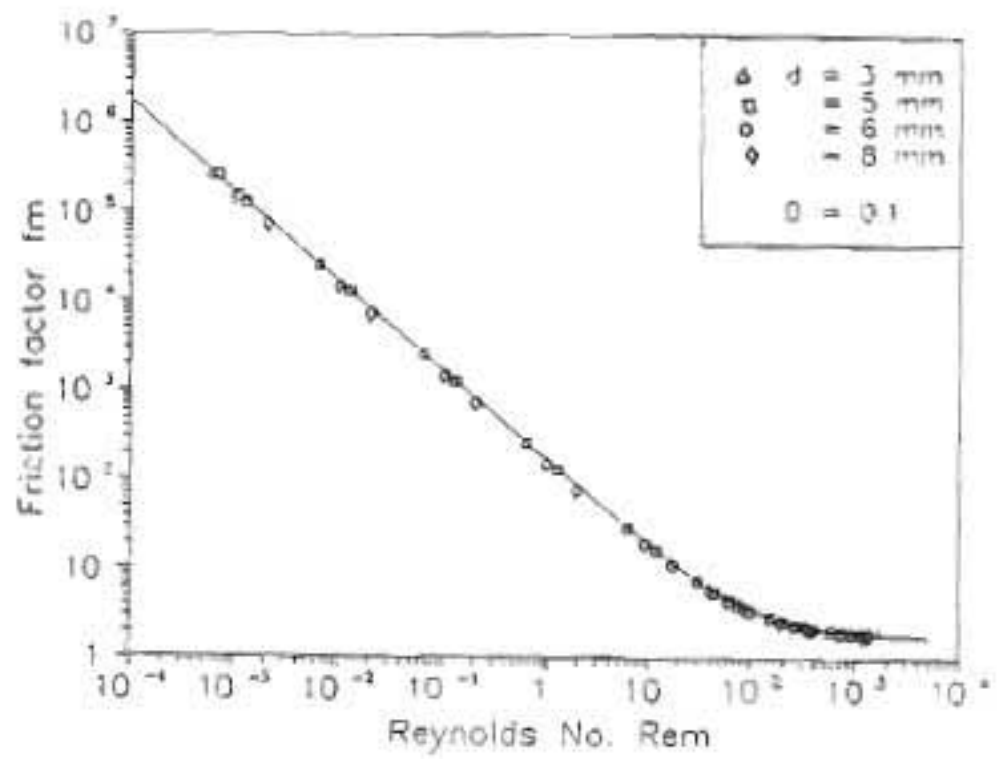

Fig. 16 total friction factor $f_{m}$ variation with $\mathrm{Re}_{\mathrm{m}}$ for $D=0.1$ and $d=3,5,6$ and $8 \mathrm{~mm}$

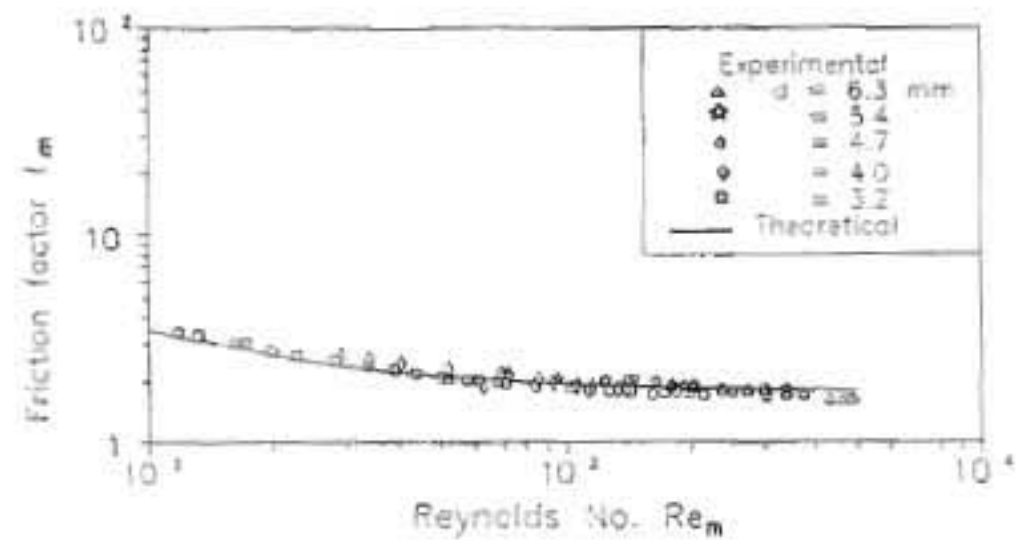

Fig.17 Total friction factor $f_{m}$ versus Reynolds number. Comparison with the experimental results 
characteristics in a circular pipe filled with porous material. The followng conclusions can be made.

The variable porosity effect appears clearly in the regions aear the walls only. It gives an increase in the velocity and signifies the channeling effect. Both the velocity and the channeling effect increase with the increase of the bead diameter, the ratio of bead diameter to channel radius $D$, or the increase of the nondimensional pressure gradient B

The soudimensional pressure gradient B slows a laear relation in a logarithmic scale with Reynolds number Re. B mereases with the increase of Re and decreases with the increase of either the bead diameter $f$ or the ratio $D$ at constant Reynolds number

The total friction $f$ curve tukes the straight line shape in the laminar region. curved stape in the transient and coustant shape for the turbulent regious. Its value decreases with the increase of the bead diameter, Re and the ratio of bead diameter to pipe rodius $D$.

The inertia frictiou factor $f_{i}$ is independent ou $\mathrm{Re}_{\mathrm{f}}$, while the Darcy friction factor $f_{D}$ changes linearly is the logaridhinic graph with $R_{e}$. The curve of $f_{1}$ assymptotes with the lines of $/ 0$ in the fiuearly shape laminar region where both the friction factors $/ \mathrm{r} / \mathrm{t}$ and $/ \mathrm{V} / \mathrm{f}_{\mathrm{t}}$ are nearly constants and very small while the Dorcy friction $f_{0} f_{f}$ coutributes the maia part of the total friction. The curve of $f_{t}$ assyuptotes with the line of $f_{f}$ for constaot value region and both the Darcy and viscous friction $/ \mathrm{C} / \mathrm{f}$. $f V / f$ can be aeglected, while the inertia friction $/ f f$, forms the main part of the torai friction.

Ergun equatiou for the non-spherical particles porous media is also valid for porous media of ideutical spherical particles for the laminar, transicnt and tubulent regimes when both Reynolds aumber and total fiction factor are based on the area mean porosity $\varepsilon_{\mathrm{u}}$ instead of the free porosity $\varepsilon_{\mathrm{c}}$

\section{Nomenclature}

A Forschbeimer inertia coefficiebt of the porous medium, equation 2

b. $c$ coastants, equation 5

B nondimensional pressure gradient, equation 7

C 1 dimensioaless coefficients, equation ?

d shere diameter, um

D dimensiouless sphere diameter $=d / r_{0}$

Da modified Darcy number $-\gamma_{m} /\left(4 r_{0}{ }^{2}, \varepsilon_{m}\right)$

fo frictional drag factor induced by the solid matrix ( Darcys pressure

drop and the flow ieertia drag) 


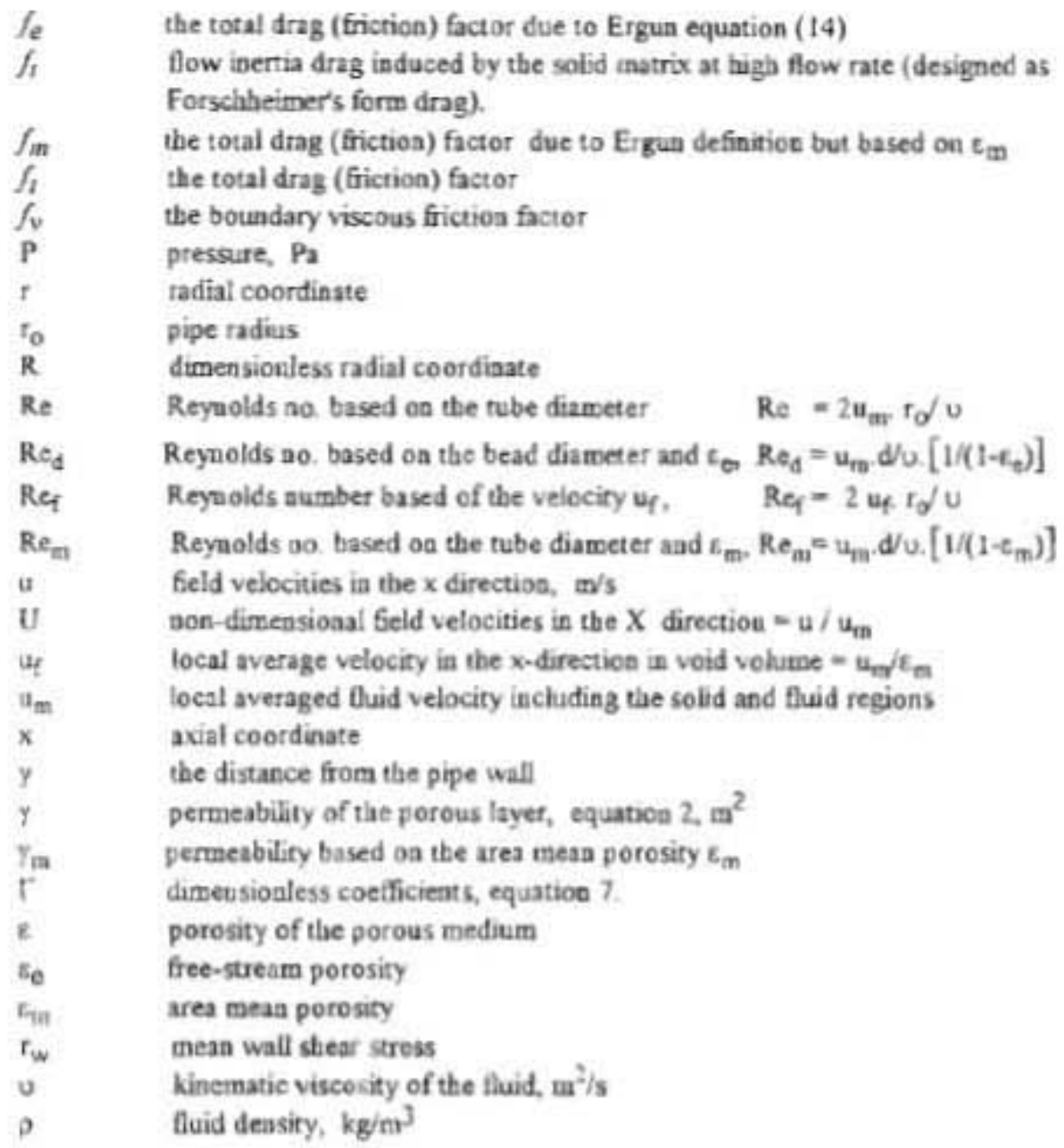

\section{References}

1. Vafia, K. and Tien, C. L. "Boundary and Lertia Effiects on Flow and Heat Transfer in Porous Media," Int. I. Heat Mass Transfer, Vol. 24, pp. 105-203, 1981.

2. Vafia, K. "Coevective Flow and Heat Tranafer a Variable porosiry Media," I. of Fluid Mechanics, Vol 147, pp, 233-259, 1984

3. Vafia, K. Alkire, R. L, and Tien, C. L." "An Experimental teveatigation of Heat Trunsfer in Variable Porosity Media," ASME Jounal of Heat Transfer, Vol 107. pp. $642-647,1985$

4. Kavany, M. "Laminat Flow Through a Porous Channel Bounded by Isothermal Parallel Plates," tat. J of Heat and Mass Transfer, Vol 28, pp. 351-353. 1985. 
5. Nakayama, A. Koyama, H., and Kuwaha, F., "An Analysis of Forced Courvection ia a Chanuel Filled With a Brinkman-Darcy Porous Medjum; Exact and Approximation Solutions," Wirme und Stoffibertragung, Vol. 23, pp. 291-295, 1938.

6. Poulikakos, D., and Renken, K, "Forced Convection in a Channel Filled With Porous Medium, Including the Effects of Flow Inertia, vanable Porosiry, and Brinkman Friction," ASME, Journal of Heat Transfer, Vol. 109, pp 880-888, 1987

7 El Kady, M. "Forced Convection Heat Transfer and Flow la an Annular Porous Medium is the Non-Darcian Effects" Mansoura Engineering Journal (MEJ), Vol. 19. No. 4, December 1994, pp.

8 Cheng. P, Hsu, C. T., and Chowdlury, A, "Forced Convection in the Entrance Region of a Packed Chanel With Asymmetric Hearing, ASME Journal of Heat Transfer, Vol. I10, pp. 946-954, 1988.

9 Bencoati, R. F, and Brosilow, C. B., "Void fraction distribution io Packed Beds," AICHE J., Vol 8, pp. 359-361, 1962.

10. Chandraseibara, B C., and Vortmeyer, D., "Flow Model for Velocity Distribution in Fixed Porous Beds Under Isothermal Couditions," Th. Fuid Dysamics, Vol 12. pp. 105-111, 1979.

11. Wang, B. and Du, J., "Forces convective beat transfer in a vertical anoulus filled with porous media" Int. J, of Heat and Mass Transfer, Vol. 36, No 17, pp. 4207. 4213, 1993

12. Amin, $\mathrm{A}$ and Vafi. K. "Analysis of dispersion effects and noe-thermal equitin rium, not-Darcios, variable porosity incompressible flow through porous media "Int. I. of Heat and Mass Trussfer, Vol. 37, No. 6. pp. 939-954, 1994

13 Ergun, S. "Fluid flow through packed columns" Chemical Enginearing Progress, Vol 48, pp. 89.94, 1952.

14. Clicag, P." Heat Transfer in Geothermal Systems," Advances in Heat Tranisfer, Vol. 14. pp. T-105. 1979

15 Muralidhar, K, and Kulacki, F. A." "Non-Darcy Naturat Convection in a Sarurated Horizontal Porous Aanulus," ASME Heat Transfer Division, HTD VoL. 56 "Natural Coavection in Porous Media", pp. 23-31, 1987

16. Chou, F. and Hwang, G. "Vorticity-Velocity method for the Graetz problem and the effect of uanural coavection in a horizontal rectungular chanael with uniform wall heat flux" ASME J. of Heat Transfer, Vol. 109, pp. 704-710, 1987

17. Hwang, G. and Chao, C., "Effects of wall conduction and Darcy Number on laminar mixed convection ia a horizontal square porous channei" ASME I. of Heat Transfer, Vol. 114, pp. 614-621, 1992.

13. Hirsch. C. "Numerical Computation of Internal and Exteral Flows, VoL 1: Fundanentals of Numerical Discretization" Jolan Wiley \& Sons, 1991.

19. Patankar, S. "Numerical Heat "Transfer and Fluid Flow" Mc Graw Hill. New York, 1980

20. Rabie, L. H. Arid, F. F, and Shalaby, M. A., "Flow of Dilute polymer solutious in perous media Mansuura Engineeriag Journal, MEI Vol. II, No. 2, December 1986 